\title{
Análisis de cobertura de redes basadas en el estándar 802.15.4
}

\author{
Luis Leonardo Camargo-Ariza ${ }^{1} \quad$ Rafael David Linero-Ramos ${ }^{2} \quad$ Byron Medina-Delgado $^{3}$
}

Recibido:

Febrero 2 de 2013

Aceptado:

Mayo 28 de 2013

\section{Resumen}

El artículo presenta un análisis de las pérdidas de señal en redes inalámbricas soportadas en el estándar 802.15.4, este análisis se basa en el concepto de balance de potencia, el modelo simplificado de pérdidas y las funciones de distribución de probabilidad de Gauss y Rayleigh. El estudio se realiza a un conjunto de medidas de intensidad de señal recibida, tomadas en una red de prueba en la banda de frecuencia de $2400 \mathrm{MHz}$, en un ambiente tipo oficina, con el objetivo de determinar modelos matemáticos que describan las pérdidas de señal y, con éstos poder realizar la correcta planificación de este tipo de redes al determinar el radio de cobertura de un transmisor.

Palabras clave: modelos, pérdidas de señal, telecomunicación, ZigBee.

\section{Abstract}

The article presents an analysis of path loss on wireless networks based on the 802.15.4 standard; this analysis is based on the concept of balance of power, the simplified path loss model, and statistical models for characterize the attenuation (Gauss shadowing and Rayleigh fading). The study is conducted to a set of measures of received signal strength, the measures are taken in a test network in the frequency band of $2400 \mathrm{MHz}$ in an office-type environment. The objective of analysis is to determine the mathematical models describing path loss for defining coverage area and make a proper planning of these networks. 
No. 1

Enero-Junio 2013 ISSN $0122-820 \mathrm{X}$

\section{Introducción}

E

estándar IEEE 802.15.4 define protocolo y compatibilidad de interconexión para dispositivos que utilizan baja velocidad de datos, transmisores de baja potencia y baja complejidad en la comunicación por radio frecuencia en Redes Inalámbricas de Área Personal (Wireless Personal Area Network, WPAN). La arquitectura del estándar está definida por dos capas: la Capa Física (Physical Layers, PHY), que especifica las frecuencias de radio que se utilizan para los enlaces y los mecanismos de control de bajo nivel y, la capa de Control de Acceso al Medio (Medium Access Control, MAC) que maneja el control de acceso al canal de radio físico [1].

ZigBee es la tecnología basada en el estándar para redes inalámbricas IEEE 802.15.4, diseñada para satisfacer las necesidades de los sensores inalámbricos con baja velocidad de trasferencia de datos [2]; esta tecnología es utilizada en aplicaciones industriales, domóticas y médicas, por ser de fácil implementación y porque requiere poca energía para funcionar [3].

En los sistemas de comunicación inalámbricos basados en ZigBee se puede presentar ruido, pérdidas y retardo de la señal. El ruido es ocasionado por otros dispositivos que transmiten en las bandas de frecuencia ISM (Industrial, Científica y Médica) [4]. Las pérdidas y retardos se deben a fenómenos de propagación de la señal, obstáculos encontrados en el medio y, recepción de la señal proveniente de múltiples trayectos generados por la reflexión y difracción de la misma [5].

Periódicamente el grupo de soporte 802.15.4a del Instituto de Ingenieros Eléctricos y Electrónicos (Institute of Electrical and Electronics Engineers, IEEE) realiza recomendaciones sobre el modelado de las pérdidas de señal en el canal de comunicaciones, en las bandas de frecuencia del estándar IEEE 802.15.4 [6]. Los modelos de pérdidas básicamente están definidos en función de la distancia, la frecuencia y el entorno [7], [8].

Para evaluar las pérdidas de la señal se implementa una red de prueba y se realizan medidas de la intensidad de señal recibida (Received Signal Strength Indication, RSSI), siendo esta variable un indicador de la calidad del enlace inalámbrico [9]. Estas medidas son realizadas con el objetivo de determinar el radio de cobertura de un transmisor ZigBee para establecer la máxima separación de los sensores en la red.

\section{Materiales y métodos}

Los dispositivos de prueba utilizados para conformar la red 802.15.4 a $2400 \mathrm{MHz}$ son XBee Series 2 modelo XB24-B del fabricante Digi International; estos dispositivos se configuraron por medio del software X-CTU Versión 5.1.4.1 del mismo fabricante, a través de una tarjeta de interfaz XBee Xplorer conectada por cable USB (Universal Serial Bus) a un computador.

El entorno de mediciones es un ambiente interior tipo oficina con equipos de cómputo, escritorios y sillas (ver Figura 1); y con la influencia de Redes de Acceso Local (Local Area Network, LAN) IEEE 802.11.

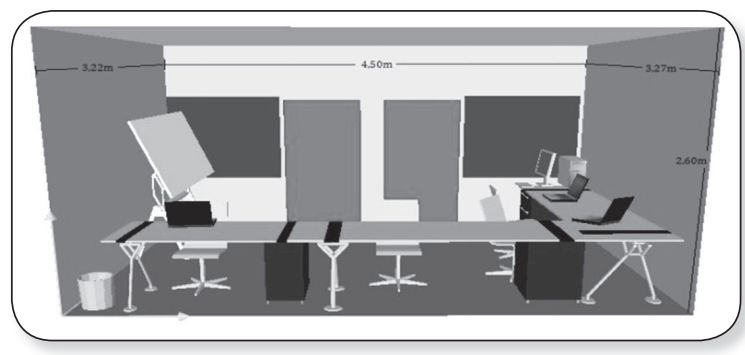

Figura 1. Escenario de mediciones.

Fuente: Instalaciones GIDEAM.

Se realizaron medidas de RSSI, en una red punto a punto con un dispositivo programado como coordinador y otro como dispositivo final, como se muestra en la Figura 2. Se 
configuró la máxima potencia de salida del transmisor $(3 \mathrm{dBm})$ y antena integrada tipo chip $(-1,5 \mathrm{dBi})$ para el receptor y el transmisor [10].

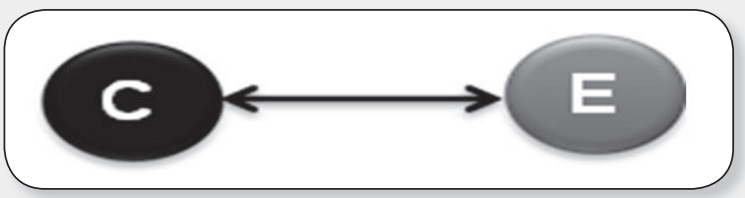

Figura 2. Red entre coordinador y dispositivo final. Fuente: Red de prueba GIDEAM.

Las medidas se inician a 0,25 metros de distancia entre el dispositivo coordinador y el dispositivo final, porque a partir de ésta empieza a variar la RSSI en función de la distancia, seguido de 1 metro, y posteriormente con incrementos de 1 metro ( $2 \mathrm{~m}, 3 \mathrm{~m}, 4 \mathrm{~m}$, etc.) hasta que la RSSI disminuya a valores cercanos a la sensibilidad del receptor $(-96 \mathrm{dBm})$; estas medidas se realizan a diferentes velocidades de transferencia de interfaz serial $(9600$ bits/s, 38400 bits/s y 115200 bits/s). Para cada alteración de las variables independientes se tomaron 10 medidas de RSSI, directamente del XBee utilizando el software X-CTU.

A partir de las medidas RSSI se calculan las pérdidas de propagación utilizando el balance de potencia, según (1).

$$
P_{R}=P_{T}+G_{T}+G_{R}-L
$$

En donde $P_{R}$ representa la intensidad de la señal recibida (RSSI), $P_{T}$ la intensidad de la señal transmitida $(3 \mathrm{dBm}), \mathrm{G}_{\mathrm{T}}$ la ganancia de la antena del transmisor, $(-1,5 \mathrm{dBi}), \mathrm{G}_{R}$ la ganancia de la antena del receptor y $L$ las pérdidas de propagación.

Con los datos obtenidos se procede a modelar las pérdidas de dicha red para cada una de las velocidades de transferencia de interfaz serial en el software de análisis estadístico y predictivo Statistica de StatSoft [11].

Los modelos empíricos de pérdidas propuestos se ajustan al modelo simplificado de pérdidas de (2) [12], en donde el parámetro $K$ representa las pérdidas iniciales, el parámetro $n$ un factor función de la frecuencia y las características del medio y, el parámetro $d$ la distancia.

$$
L=K+10 n \log \left(\frac{d}{d_{0}}\right)
$$

Para el ajuste se realiza una transformación logarítmica de la variable independiente $(d / 0,25)$, a partir de ésta se obtienen los modelos utilizando regresiones simples. Además, se evalúa la discrepancia entre los valores observados y los valores esperados en el modelo de estudio, midiendo la bondad del ajuste con el coeficiente de determinación $R^{2}$ [13].

Para validar el modelo obtenido es necesario que las diferencias entre el valor medido de la variable dependiente y el valor obtenido con el modelo cumplan los siguientes requisitos: deben presentar una distribución normal, que se comprueba a través del test de Shapiro-Wilk y KolmogorovSmirnov con la corrección de Lilliefors; la varianza de los mismos debe ser constante, es decir, debe existir homocedasticidad; no debe existir autocorrelación entre éstos, lo cual se comprueba a través del contraste de DurbinWatson; y en el caso de regresión múltiple entre las variables independientes no debe existir relación [14].

Después de obtener y validar los modelos, se estima el radio de cobertura de la red a partir de las pérdidas, considerando una probabilidad de interrupción del 1\%, siguiendo la distribución de Gauss. El cálculo del radio de cobertura $(d)$ se realiza a partir de (1) y (3) [15].

$$
L=K+10 n \log \left(\frac{d}{d_{0}}\right)+\varphi_{d B}
$$

En donde $\varphi_{d B}$ es la ganancia relativa de pérdidas medidas, este valor depende de la probabilidad según la distribución gaussiana, con media $\mu_{\text {dגB }}$ igual a cero y desviación estándar. Ver (4) y Figura 3.
Enero-Junio 2013 ISSN 0122-820X 
No. 1

Enero-Junio 2013

ISSN 0122-820X $\sigma_{\varphi_{d B}}=\sqrt{\frac{1}{N} \sum_{i=1}^{N}\left[L_{\text {medida }}\left(d_{i}\right)-L_{\text {moladad }}\left(d_{i}\right)\right]^{2}}$

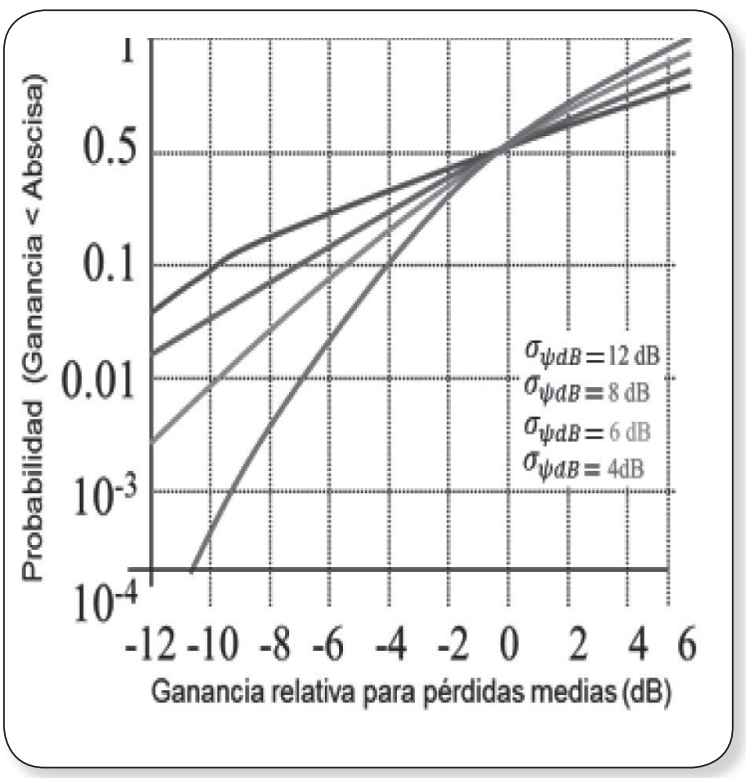

Figura 3. Función de distribución de probabilidad de Gauss.

Fuente: Modern Wireless Communications [16].

La desviación estándar $\sigma_{\psi d B}$ se calculó para cada modelo por medio de (5).

$\sigma_{\varphi_{d B}}=\sqrt{\frac{1}{N} \sum_{i=1}^{N}\left[L_{\text {medida }}\left(d_{i}\right)-L_{\text {moladad }}\left(d_{i}\right)\right]^{2}}$

En donde $N$ representa el número de muestras, $L_{\text {medida }}$ son las pérdidas medidas, y $L$ ${ }_{d a}$ son las pérdidas calculadas con el modelo.

12

El radio de cobertura (d) calculado es comparado con el radio obtenido, si el margen de requerimiento $\varphi_{d B}$ es deducido con la distribución de desvanecimiento de Rayleigh, distribución que describe la variación estadística de la envolvente de la señal resultante de la propagación en múltiples trayectos [16]. Ver (6) y (7) y, Figura 4.

$$
\operatorname{Prob}(v<V)=\int \frac{v}{\sigma^{2}} e^{-\frac{v^{2}}{2 \sigma^{2}}} d v=1-e^{-\frac{V^{2}}{2 \sigma^{2}}}
$$

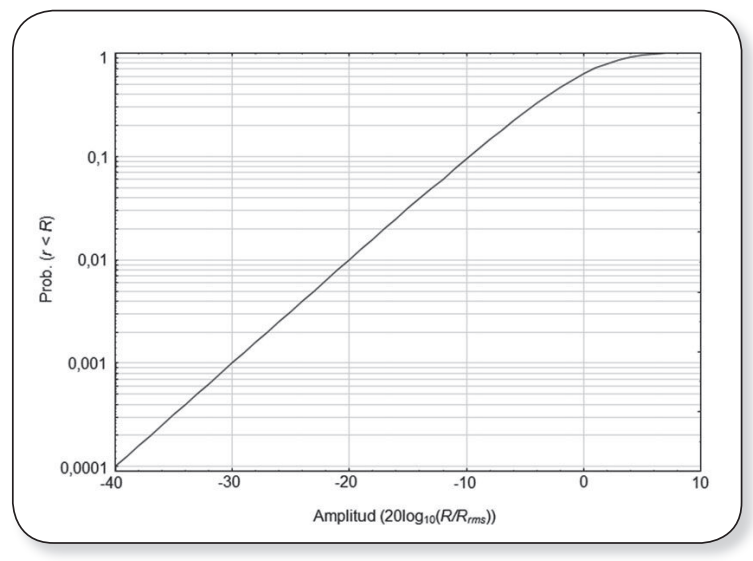

Figura 4. Función de distribución de probabilidad de Rayleigh.

Fuente: Modern Wireless Communications [16].

$$
\varphi_{d B}=20 \log \left(\frac{V}{V_{0_{r m s}}}\right)
$$

En donde $V$ es la amplitud del voltaje de la envolvente compleja desvanecida en el trayecto y $\sigma^{2}=P_{0} / 2$, entonces el valor eficaz de $V_{0}$ es $V_{0}=(2)^{1 / 2} \sigma$.

\section{Resultados y discusión}

Se realiza un total de 1680 medidas de RSSI (ver Figura 5).

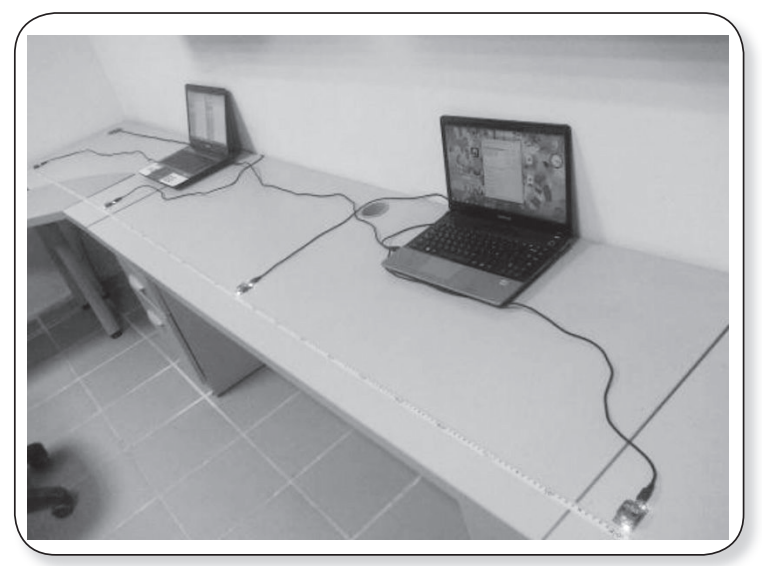

Figura 5. Foto de una jornada de medidas.

Fuente: Instalaciones GIDEAM.

Con respecto a las medidas de RSSI, se observa que al aumentar la distancia entre los dispositivos el RSSI disminuye debido al 
aumento de pérdidas en el canal; estas pérdidas fueron modeladas para cada una de las velocidades de transferencia de interfaz serial a través de regresiones simples obteniendo los modelos relacionados a continuación.

Para una velocidad de transferencia de interfaz serial de 9600 bps, ver (8).

$$
\begin{gathered}
L=46,53+10(2,69405) \log \left(\frac{d}{0,25}\right) \\
0,25 m \leq d \leq 6 m
\end{gathered}
$$

Este modelo tiene un $R^{2}=0,91421520$, sustentando que las variaciones observadas en las pérdidas de señal se deben en el 91,42\% de los casos, a los cambios en la distancia de separación de los dispositivos en la red, lo que hace a este un modelo significativo. Sus residuos presentan una distribución normal, que se comprueba a través del test de Shapiro-Wilk $(W=0,95501, p>0,11285)$ y Kolmogorov-Smirnov K-S $(d=0,11072$, $p>0,2)$, con la corrección de Lilliefors $(p>0,2)$; existe homocedasticidad, y no existe autocorrelación entre éstos, lo cual se comprueba a través del contraste de DurbinWatson $(d=1,902137$ para $n=560)$, con un nivel de significación de $\sigma=0,05$. Ver Figura 6.

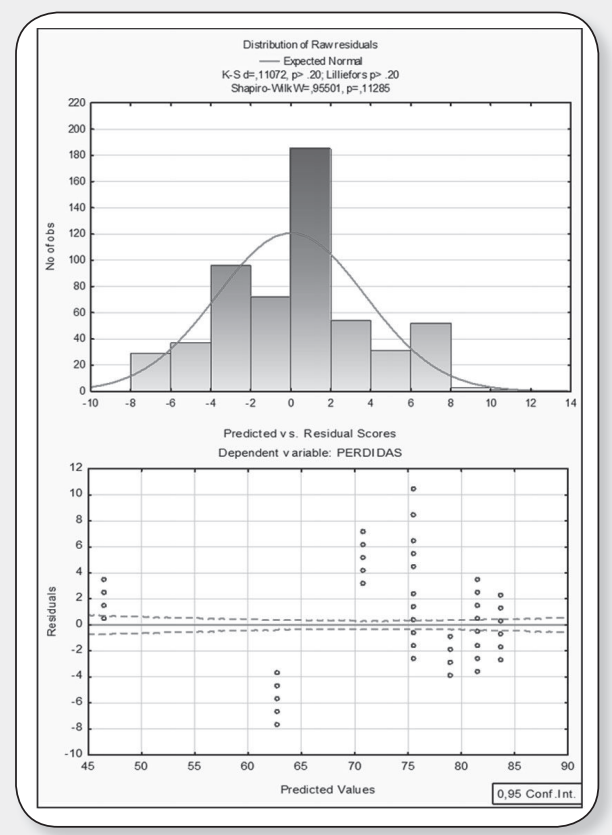

Figura 6. Validación del modelo a 9600 bps.

Fuente: Software Statistica [11].
Para una velocidad de transferencia de interfaz serial de 38400 bps, ver (9).

$$
\begin{gathered}
L=53,2277+10(2,49769) \log \left(\frac{d}{0,25}\right) \\
0,25 m \leq d \leq 6 m
\end{gathered}
$$

Este modelo es significativo, tiene un $R^{2}=$ 0,86636426 , sustentando que las variaciones observadas en las pérdidas de señal se deben en el $86,63 \%$ de los casos, a los cambios en la distancia de separación de los dispositivos. Sus residuos presentan una distribución normal, que se comprueba a través del test de Shapiro-Wilk $(W=0,97351, p>0,23175)$ y Kolmogorov-Smirnov K-S ( $d=0,09809$, p > 0,2), con la corrección de Lilliefors $(p>0,2)$; existe homocedasticidad, y no existe autocorrelación entre éstos, lo cual se comprueba a través del contraste de DurbinWatson $(d=1,913271$ para $n=560)$, con un nivel de significación de $\sigma=0,05$. Ver Figura 7 .

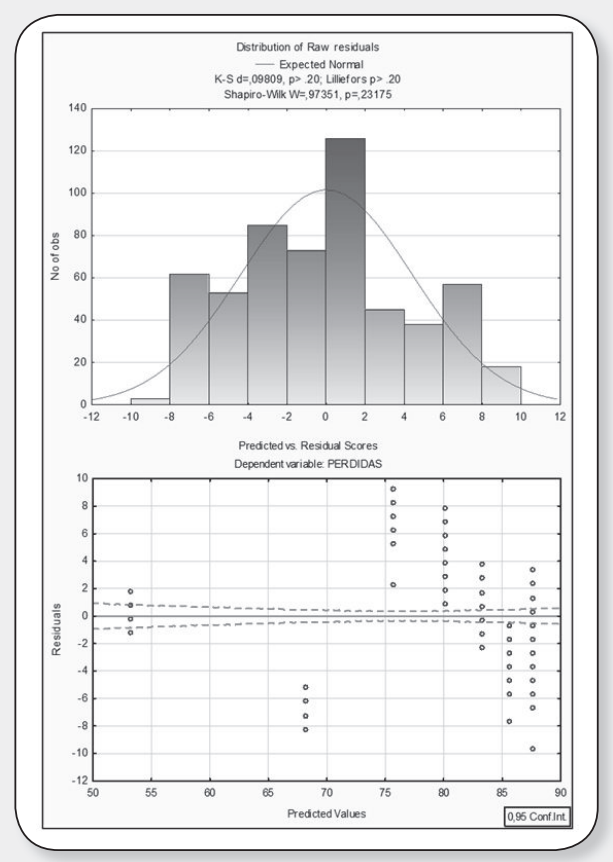

Figura 7. Validación del modelo a 38400 bps.

Fuente: Software Statistica [11]. SSN 0122-820X

Para una velocidad de transferencia de interfaz serial de 115200 bps, ver (10).

$$
\begin{gathered}
L=51,3431+10(2,75667) \log \left(\frac{d}{0,25}\right) \\
0,25 m \leq d \leq 6 m
\end{gathered}
$$


No. 1

Enero-Junio 2013 ISSN 0122-820X
Este modelo también es significativo debido a que $R^{2}=0,89319642$, esto expresa que los cambios en la distancia de separación de los dispositivos explican en el 89,31\% de los casos, las pérdidas de RSSI. Los residuos presentan una distribución normal, lo cual se comprueba a través del test de Shapiro-Wilk $(W=0,96285, p>0,14425)$ y KolmogorovSmirnov K-S $(d=0,10025, p>0,2)$, con la corrección de Lilliefors $(p>0,2)$; existe homocedasticidad, y no existe autocorrelación entre éstos, lo cual se comprueba a través del contraste de Durbin-Watson $(d=1,9374823$ para $n=560$ ), con un nivel de significación de $\sigma=0,05$. Ver figura 8 .

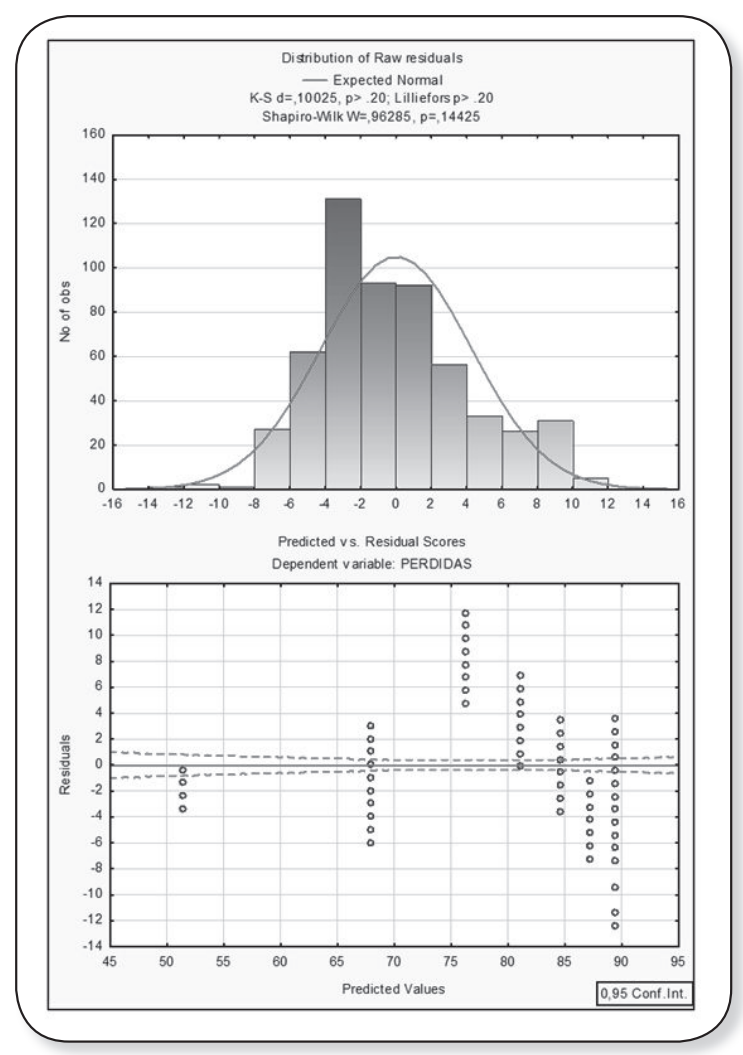

Figura 8. Validación del modelo a 115200 bps.

Fuente: Software Statistica [11].

En la figura 9 se observa el comportamiento de las pérdidas en función de la distancia entre dispositivos, según los modelos para cada una de las velocidades de transferencia de interfaz serial configuradas.

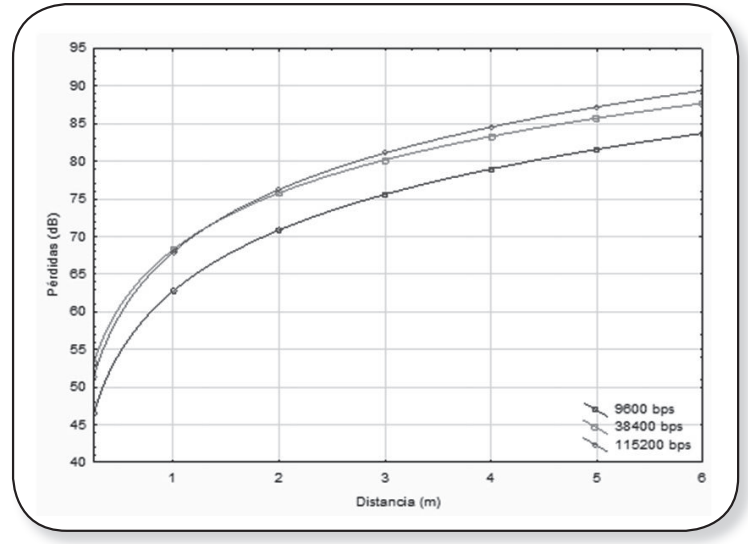

Figura 9. Modelos de pérdidas.

Fuente: Software Statistica [11].

Estos modelos se asemejan a los modelos realizados por el subgrupo de trabajo IEEE 802.15.4a; como se aprecia en la tabla 1 [6].

Tabla 1. Parámetros modelo IEEE 802.15.4a.

\begin{tabular}{|l|c|c|}
\hline \multirow{2}{*}{\multicolumn{1}{|c|}{ Entorno }} & \multicolumn{2}{c|}{ Parámetro } \\
\cline { 2 - 3 } & $\mathbf{K}$ & $\mathbf{n}$ \\
\hline Residencial LOS & 43,9 & 1,79 \\
\hline Residencial NLOS & 48,7 & 4,58 \\
\hline Oficina LOS & 36,6 & 1,63 \\
\hline Oficina NLOS & 51,4 & 3,07 \\
\hline Industrial LOS & 56,7 & 1,2 \\
\hline Industrial NLOS & 56,7 & 2,15 \\
\hline
\end{tabular}

Fuente: IEEE 802.15.4a channel model-final report [6].

En los modelos obtenidos se observa que al aumentar la velocidad de transferencia de interfaz serial las pérdidas se incrementan, por lo tanto, se genera un nuevo modelo a través de regresión múltiple, con el fin de evaluar si esta variable explica significativamente la variabilidad de las pérdidas. Ver (11), en donde $B R$ es la velocidad de la interfaz serial.

$$
\begin{aligned}
L= & 27,0678+5,1289 \log (B R)+ \\
& 10(2,64947) \log \left(\frac{d}{0,25}\right)
\end{aligned}
$$

Este modelo es significativo con un coeficiente de terminación $R^{2}=0,889743967$, las 
variables independientes explican el $88,97 \%$ de las variaciones en las pérdidas; el logaritmo de la distancia describe el 0,858614 (85,86\%) de las pérdidas y el logaritmo de $B R$ un 0,031130 (3,11\%) de las pérdidas. Además, las variables independientes tienen tolerancia de 1 , valor superior a 0,5 determinando que no existe colinealidad entre éstas.

Los residuos del modelo siguen una distribución normal, esto se valida con el test de Shapiro-Wilk $(W=0,9852, p>0,3664)$ y Kolmogorov-Smirnov K-S $(d=0,2186, p>$ $0,2)$, con la corrección de Lilliefors $(p>0,2)$; la varianza de los residuos es constante, presentando homocedasticidad. Ver Figura 10.

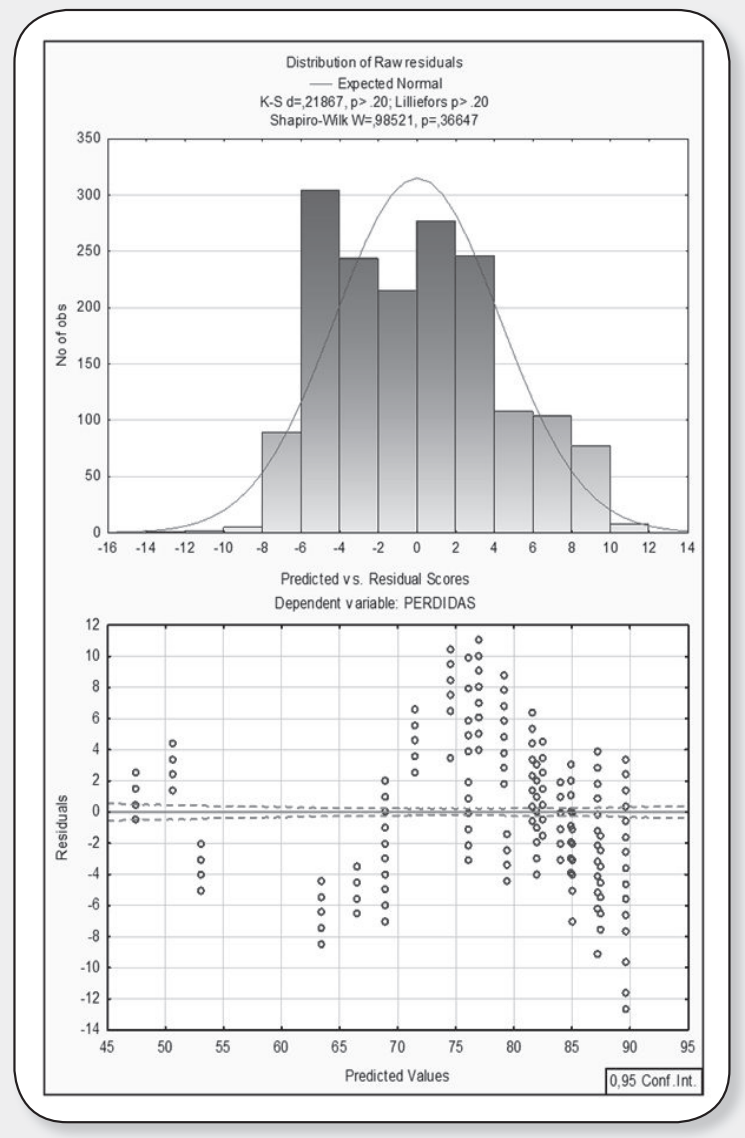

Figura 10. Validación del modelo general. Fuente: Software Statistica [11].

Con base en los modelos obtenidos se calculan los radios de cobertura de la red modelada, con un requerimiento del $99 \%$ de disponibilidad, para una sensibilidad del receptor de $-96 \mathrm{dBm}$ (Ver Figura 11).

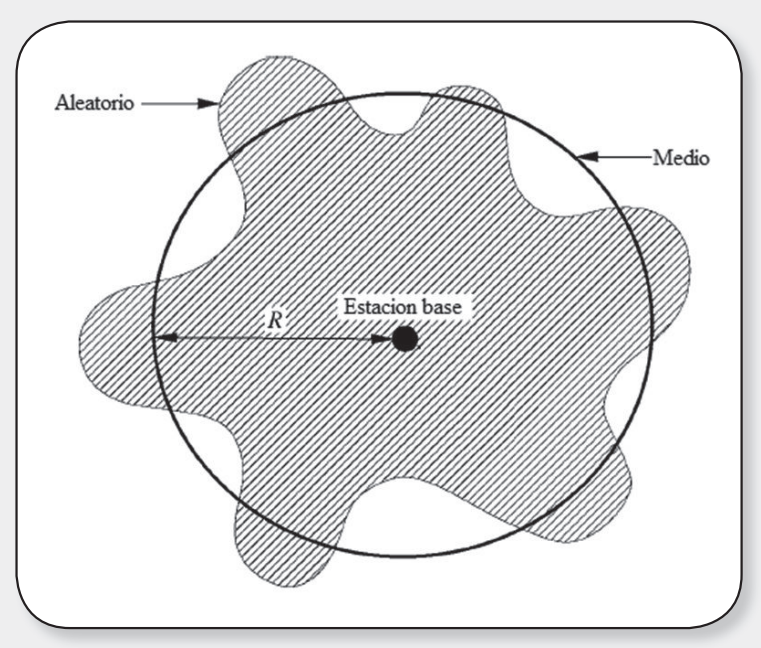

Figura 11. Radio de cobertura.

Fuente: Red de prueba GIDEAM.

Para la velocidad de transferencia de interfaz serial de 9600 bps, la desviación estándar de la atenuación del canal es de 3,70 $d B$, si es requerido un $99 \%$ de disponibilidad, se necesita un extra de $6 \mathrm{~dB}$ de margen según la distribución de Gauss y un margen de 20 $d B$ según la distribución de Rayleigh, ver figuras 3 y 4; por consiguiente la potencia de señal media al margen del límite de cobertura debe ser $-90 \mathrm{dBm}$ y $-76 \mathrm{dBm}$, y no $-96 \mathrm{dBm}$ (sensibilidad del Rx). A partir de esto se calcula el radio de cobertura $d(d=10,27 \mathrm{~m}$ y $d=3,1 \mathrm{~m}$ ), de acuerdo con (1), (2) y (8).

El radio calculado a partir de la distribución de Rayleigh sugiere un desvanecimiento rápido de la señal a una probabilidad de $1 \%$ de interrupción, por esto se realizan los cálculos con una probabilidad de interrupción del 10\%. Para 9600 bps a 10\% de interrupción, el radio es de 7,44 m.

Para la velocidad de transferencia de interfaz serial de 38400 bps la desviación estándar es de 4,40 dB, el radio calculado a partir de la distribución de Gauss con una probabilidad de interrupción de $1 \%$ es de $6,76 \mathrm{~m}$; y el radio calculado a partir de la distribución de Rayleigh con una probabilidad de interrupción de $10 \%$ es de $5,23 \mathrm{~m}$. 
No. 1

Enero-Junio 2013 ISSN $0122-820 \mathrm{X}$
Para la velocidad de transferencia de interfaz serial de 115200 bps la desviación estándar es de 4,28 dB, el radio calculado a partir de la distribución de Gauss con una probabilidad de interrupción de $1 \%$ es de $5,9 \mathrm{~m}$; y el radio calculado a partir de la distribución de Rayleigh con una probabilidad de interrupción de $10 \%$ es de 4,6 m.

\section{Conclusiones}

En una red de dispositivos XBee Series 2 el tamaño de los paquetes de datos enviados no afecta las pérdidas que se presentan en el sistema, pero la distancia entre dispositivos y la velocidad de transferencia afectan significativamente el valor de la intensidad de la señal recibida.

El radio de cobertura máximo de una red de dispositivos XBee Series 2 en un entorno de oficina y con una disponibilidad del 99\% e independientemente de la velocidad de transferencia serial es aproximadamente de $6 \mathrm{~m}$.

\section{Referencias}

[1] IEEE Standard for Local and metropolitan area networks Part 15.4: Low-Rate Wireless Personal Area Networks (LR-WPANs), IEEE Std 802.15.4-2011, 2011.

[2] Z. Alliance, "ZigBee Technology." [Online]. Available: www.zigbee. networks with other RF devices at home", Sens. Technol. ..., pp. 200-205, Jun. 2009.

[5] A. Sikora and V. Groza, "Coexistence of IEEE802. 15.4 withother Systems in the 2.4 GHz-ISM-Band", ..., 2005. IMTC 2005. Proc. ..., pp. 1786-1791, 2005.

[6] A. F. Molisch, K. Balakrishnan, C. Chong, S. Emami, A. Fort, J. Karedal, J. Kunisch, H. Schantz, U. Schuster, and K. Siwiak, "IEEE 802.15.4a channel model-final report", 2004.

[7] G. Kadel and R. Lorenz, "Impact of the radio channel on the performance of digital mobile communication systems", in Sixth IEEE Int. Symp. on Personal, Indoor and Mobile Radio Communications PIMRC'95, pp. 419-423, 1995.

[8] J. P. Rossi, "Influence of measurement conditions on the evaluation of some radio channel parameters", IEEE Trans. on Vehicular Technology, vol. VT-48, pp. 1304-1316, July 1999.

[9] K. Benkic and M. Malajner, "Using RSSI value for distance estimation in wireless sensor networks based on ZigBee”, Syst. Signals ..., 2008.

[10] D. International, "XBee $®$ / XBeePRO @ ZB RF Modules Digi International Inc.", 2010. [Online]. Available: http://ftp1.digi.com/support/ documentation/90000976_P.pdf. [Accessed: 23-Jan-2013].

ZigBeeTechnology.aspx. [Accessed: 15Jan-2013].

[3] C. Gómez, J. Paradells, and J. E. Caballero, Sensors Everywhere Wireless Network Technologies and Solutions. Fundación Vodafone España, 2010.

[4] H. Huo, Y. Xu, C. Bilen, and H. Zhang, "Coexistence issues of $2.4 \mathrm{GHz}$ sensor
[11] StatSoft, Inc. (2013). Statistica (data analysis software system), version 12.www.statsoft.com.

[12] V. Erceg, L. J. Greenstein, S. Y. Tjandra, S. R. Parkoff, A. Gupta, B. Kulic, A. A. Julius, and R. Bianchi, "An empirically based path loss model for wireless channels in suburban environments", IEEE J. Sel. AreasCommun., pp. 1205- 
1211, July 1999.

[13] J. L. Devore, Probabilidad y Estadística para Ingeniería y Ciencias. Cengage Learning Editores, 2008, pp. 500-549.

[14] C. Guisande, A. Vaamonde, y A. Barreiro, Tratamiento de datos con $R$, STATISTICA y SPSS. Ediciones Díaz de Santos, 2011.

[15] A. Goldsmith, Wireless Communications. Cambridge: Cambridge UniversityPress, 2005, pp. 27-63.

[16] S. Haykin and M. Moher, Modern Wireless Communications. Pearson Prentice Hall, 2005. 